\title{
Entropy-Based, Multiple-Portal-to-3DCT Registration for Prostate Radiotherapy Using Iteratively Estimated Segmentation
}

\author{
Ravi Bansal ${ }^{1}$, Lawrence H. Staib ${ }^{1}$, Zhe Chen $^{2}$, Anand Rangarajan ${ }^{1}$, Jonathan \\ Knisely $^{2}$, Ravinder Nath ${ }^{2}$, and James S. Duncan ${ }^{1}$ \\ 1 Departments of Electrical Engineering and Diagnostic Radiology \\ 2 Department of Therapeutic Radiology \\ Yale University, New Haven, CT 06520-8042
}

\begin{abstract}
In external beam radiotherapy (EBRT), patient setup verification over the entire course of fractionated treatment is necessary for accurate delivery of specified dose to the tumor. We develop an information theoretic minimax entropy registration framework for patient setup verification using portal images and the treatment planning 3D CT data set. Within this framework we propose to simultaneously and iteratively segment the portal images and register them to the 3D CT data set to achieve robust and accurate estimation of the pose parameters. Appropriate entropies are evaluated, in an iterative fashion, to segment the portal images and to find the registration parameters. Earlier, we reported our work using a single portal image to estimate the transformation parameters. In this work, we extend the algorithm to utilize dual portal images. In addition, we show the performance of the algorithm on real patient data, analyze the performance of the algorithm under different initializations and noise conditions, and note the wide range of parameters that can be estimated. We also present a coordinate descent interpretation of the proposed algorithm to further clarify the formulation.
\end{abstract}

\section{Introduction}

In external beam radiotherapy (EBRT) uncertainties due to patient setup errors can be reduced by registering the high contrast simulator images, obtained at diagnostic energies $(40-100 \mathrm{KeV})$, to the low resolution, low contrast 2D portal images, which are obtained using the treatment energy $\mathrm{X}$-rays (4-20 MeV). Two dimensional analysis of patient setup verification using single portal and simulator images is restricted to the verification of in-plane rotations and translations. Out-of-plane rotations and translations of the patient can degrade the accuracy of the image registration [13].

To account for out-of-plane rotations and translations, a pair of simulator and portal images of the same patient, obtained from different views, can be employed. However, such an analysis of the patient setup from 2D images can lead to inconsistencies in the determination of the transformation parameters [13]. 
Many treatment centers are moving towards offering full 3D conformal treatments that are initially planned from 3D CT datasets. Thus, for consistent and accurate three--dimensional analysis of the patient setup, it is necessary to register the 3D CT datasets to the 2D portal images. However, due to the poor quality of the portal images, automated registration of the portal images to the CT data set has remained a difficult task.

A number of methods have been proposed for both two dimensional and three dimensional analysis of the patient setup. Algorithms for two dimensional analysis include gray-level intensity based image alignment algorithms [3,14], visual inspection by the physician [23] and the anatomical landmark-based approaches $[5,13,20,21,22]$. Studies which carry out three-dimensional registration of the treatment planning 3D CT data set to the 2D portal images include interactive determination of patient setup [12], silhouette based techniques [18], gray scale correlation-based methods [8,19], a pattern-intensity based method [26], and a ridge-based method [11]. One of these approaches is interesting in that it also makes use of multi-scale medial information in the anatomical structure, using a strategy known as cores [10].

We have been developing an information theoretic registration framework, the initial form of which was reported in [1], where segmentation of a portal image and registration to the 3D CT data set is carried out iteratively and simultaneously. This framework is based on the intuition that if we have a rough estimate of the segmentation of the portal image, then it can help estimate the registration parameters. The estimated registration parameters can then in turn be used to better segment the portal image and so on. This framework is termed minimax entropy as it has two steps: the $\max$ step and the min step. In the entropy maximization step, the segmentation of the portal image is estimated, using the current estimates of the registration parameters. In the entropy minimization step, the registration parameters are estimated, based on the current estimates of the segmentation. The algorithm can start at any step, with some appropriate initialization on the other.

\section{Mathematical Notations and Formulation}

Let, $\mathbf{X}=\{\mathbf{x}(\mathrm{i})\}$, for $i=1, \ldots, N^{2}$ denote the $N \times N$ random field from which the portal images are sampled. Let, $\mathbf{G}=\{\mathbf{g}(\mathrm{i})\}$, for $i=1, \ldots, N^{3}$ denote the random field from which $3 \mathrm{D}$ CT images are sampled. Let $\mathbf{Y}(T)=\{\mathbf{y}(\mathrm{i}, T)\}$ for $i=1, \ldots, N^{2}$ denote the $N \times N$ random field from which the projections from the 3D CT data set are sampled, at a given set of transformation parameters $\mathbf{T}=\mathrm{T}$. The projected 3D CT images are also called the digitally reconstructed radiographs (DRRs). We will assume that the pixels for all the random fields are independently distributed. Thus, the probability density function of the random field $\mathbf{X}$ can be written in the factored form as $p_{\mathbf{X}}(X)=\prod_{i} p_{\mathbf{x}_{i}}\left(x_{i}\right)$. Note that for notational simplicity, we shall now write $\mathbf{x}(i)$ as $\mathbf{x}_{i}$ and $\mathbf{y}(i, T)$ as $\mathbf{y}_{i}$.

Segmentation information is incorporated into the problem by considering the joint density function $p\left(x_{i}, y_{i}\right)$ as a mixture density. 
Let $A=\{$ bone,no-bone $\}=\{1,2\}$, denote the set of classes into which the pixels are classified. The set of classes can also be denoted by a set of vectors, $\{(1,0),(0,1)\}$. Since $\mathrm{X}$-rays pass through both bone and soft tissue, a pixel in portal image is classified as bone if the $\mathrm{X}$-ray passes through at-least some bone tissue, rest of the pixels are classified no-bone. Let, $\mathbf{M}=\{\mathbf{m}(\mathrm{i})\}$, for $i=1, \ldots, N^{2}$ denote the $N \times N$ random field on the segmentation of the portal images.

Let $\mathbf{Z}$ be the $N^{2}$ x 2 classification matrix, with each row $\mathbf{z}_{i}$ of the matrix defining a set of random variables $\mathbf{z}_{i}=\left(\mathbf{z}_{1 i}, \mathbf{z}_{2 i}\right)$, defined to be:

$$
\mathbf{z}_{1 i}=\left\{\begin{array}{l}
1, \text { if } \mathbf{m}_{i}=\text { bone } \\
0, \text { if } \mathbf{m}_{i}=\text { no-bone }
\end{array}, \mathbf{z}_{2 i}=\left\{\begin{array}{l}
0, \text { if } \mathbf{m}_{i}=\text { bone } \\
1, \text { if } \mathbf{m}_{i}=\text { no-bone }
\end{array}\right.\right.
$$

The expected values of the random variables $\mathbf{z}_{a i}, \forall a$, denoted by $\left\langle z_{a i}\right\rangle=$ $P\left(\mathbf{m}_{i}=a\right)$, satisfy the constraint, $\sum_{a \in A}<z_{a i}>=1$. Note that the random variables $\mathbf{z}_{1 i}, \mathbf{z}_{2 i}$ are negatively correlated random variables, with the random variable $\mathbf{z}_{i}$ taking only two possible values, $\{(1,0),(0,1)\}$.

For clarification, we first pose our problem in a maximum a-posteriori (MAP) framework where both the segmentation, $\mathbf{M}$, and the transformation parameters, T, are being estimated explicitly. However, we note some restrictions with this approach for our problem and thus we propose a maximum likelihood (ML)/EM [7] framework to overcome these restrictions. For our problem, the EM approach has several restrictions, which lead us to propose our new minimax entropy strategy described (in section 3).

\subsection{Maximum A-Posteriori (MAP) Formulation}

An estimate of the segmentation of the portal image, $\mathbf{M}$, can be used to help estimate pose, $\mathbf{T}$, of the 3D CT data set based on a MAP formulation to simultaneously estimate the pose $\mathbf{T}$ and the portal image segmentation, $\mathbf{M}$, as follows:

$$
(\hat{T}, \hat{M})=\arg \max _{T, M} p(T, M \mid X, G)=\arg \max _{T, M} \sum_{i}\left(\sum_{a \in A} z_{a i} \ln p_{a}\left(x_{i}, y_{i}\right)-\ln p\left(y_{i}\right)\right)
$$

where we assume each pixel is statistically independent, and the joint density function, $p\left(x_{i}, y_{i}\right)$, can be written as a mixture density, in terms of component density functions, $p_{a}\left(x_{i}, y_{i}\right)$, as, $p\left(x_{i}, y_{i}\right)=\sum_{a \in A} P\left(\mathbf{m}_{i}=a\right) p\left(x_{i}, y_{i} \mid \mathbf{m}_{i}=a\right)=$ $\sum_{a \in A} P\left(\mathbf{m}_{i}=a\right) p_{a}\left(x_{i}, y_{i}\right)=\sum_{a \in A} P_{i}(a) p_{a}\left(x_{i}, y_{i}\right)$ where, $\mathbf{m}_{i}$ is the random variable denoting label at the $i$ th pixel.

This formulation requires that the algorithm solve for a unique segmentation of the portal image, $\mathbf{M}$, for a unique estimate of the pose parameters, $\mathbf{T}$. An estimated segmentation will effect the estimate of the pose parameters. Since we feel that accurate segmentation of a portal image, in general, is quite difficult, we prefer instead not to commit the algorithm to a particular segmentation.

\subsection{Maximum-Likelihood (ML) Formulation}

Thus, instead of solving the MAP problem, we pose our problem in a ML framework, with segmentation labels appearing as hidden variables. Such an approach could be captured using an EM algorithm. 
The ML/EM estimate [7] of the pose parameters can be formulated as:

$$
\hat{T}=\arg \max _{T} \ln p(T \mid X, G)=\arg \max _{T} \sum_{i}\left(\sum_{a \in A}<z_{a i}>^{k} \ln p_{a}\left(x_{i}, y_{i}\right)-\ln p\left(y_{i}\right)\right)
$$

where $<z_{a i}>^{k}=\left(\frac{<z_{a i}>^{k-1} p_{a}^{k-1}\left(x_{i}, y_{i}\right)}{\sum_{b \in A}<z_{b i}>^{k-1} p_{b}^{k-1}\left(x_{i}, y_{i}\right)}\right)$. We assume a uniform prior on the pose parameters, $\mathbf{T}$, and ignore the term $p(G \mid T)$, since the 3D CT data set, $\mathbf{G}$, is statistically independent of the pose parameters, $\mathbf{T}$.

In the ML formulation of the problem, only the transformation parameters are being estimated, with a probability distribution on the segmentation labels being estimated. This allows the algorithm to not commit to a particular segmentation of the portal image.

There are two reasons why we need to move beyond the above listed idea to find an appropriate solution to our problem. First, the EM algorithm for the mixture model as formulated above, requires that the form of $p_{a}\left(x_{i}, y_{i}\right), \forall a$ is known (i.e. one should know whether they are Gaussian, Rayleigh, exponential, etc.). For multi-modal image registration it is difficult, if not impossible, to know a priori the joint density function between the pixel intensities in the two images. Second, in the EM framework, the prior probabilities on each pixel, $P_{i}(a)$, are required to be known. If these probabilities are not known, then they can also be estimated within the EM framework, assuming that the segmentation labels on each pixel are independently and identically distributed (i.i.d.), i.e., $P_{i}(a)=\pi_{a}$, where $\pi_{a}$ satisfy the constraint $\sum_{a \in A} \pi_{a}=1$. For our problem, i.i.d. assumption does not hold.

\section{Minimax Entropy Formulation}

We overcome the restrictions of the ML formulation by borrowing the idea of averaging over the estimated density function from mutual information (MI). MI was first proposed and successfully applied for multi-modality image registration by two research groups [25,6]. The proposed minimax algorithm [2] for solving the basic problem posed by equation (2), in a computational form similar to EM, has two steps, the max step and the min step, which are evaluated iteratively to determine the registration parameters and the probability distribution of the portal image segmentation. The $\max$ step is formulated as follows:

Max Step:

$$
P^{k}(M)=\arg \max _{P(M)}\left[-\sum_{M} P(M) \ln P(M)+\sum_{M} P(M) \ln P\left(M \mid X, Y\left(T^{(k-1)}\right)\right)\right]
$$

under the constraint $\sum_{M} P(\mathbf{M}=M)=1$, where $\mathbf{M}$ is the random variable whose domain is the set of possible segmentations of the portal image, where each pixel can be labeled from the set of labels $A$. We assume that pixel labels are statistically independent, i.e., $P(\mathbf{M}=M)=\prod_{i} P\left(\mathbf{m}_{i}=a\right)=\prod_{i} P_{i}(a)$. As formulated above, the max-step simply states that the maximum entropy estimate 
of the probability $P(\mathbf{M}=M)$ is the posterior probability on the segmentation of the portal image, i.e $P\left(M \mid X, Y\left(T^{(k-1)}\right)\right)$, given the current estimate of the transformation parameters, $T^{(k-1)}$, the portal image, $X$, and the DRR, $Y$ [4]. This simple formulation of the estimated probability of a segmentation of the portal image allows us to systematically put constraints on the segmentation probability function, as we show below. The analytical solution to equation (3) estimates the probability of a segmentation label at the $i$ th pixel to be:

$$
P_{i}^{k}(a)=\left(\frac{P_{i}^{k-1}(a) p_{a}^{k-1}\left(x_{i}, y_{i}\right)}{\sum_{b \in A} P_{i}^{k-1}(b) p_{b}^{k-1}\left(x_{i}, y_{i}\right)}\right)
$$

where the component density functions, $p_{a}^{k-1}\left(x_{i}, y_{i}\right)$, are estimated from the previous step.

Note that the $P_{i}^{k}(a)$ 's, in the $k$ th iteration, form the weighing terms in the Parzen window estimates, in equation (6) below, of the component density functions, $p_{a}(x, y)$. The component density functions, in turn, are used to estimate the joint entropies, $H_{a}(x, y)=-\iint p_{a}(x, y) \ln p_{a}(x, y) d x d y$, which are minimized in the min step to estimate the registration parameters.

In order to better incorporate our initial uncertainty on the registration parameters into the problem, an annealing schedule [17] is imposed on the estimated probability of a segmentation of the portal image pixel. The modified max step, equation (3), can thus be written as:

$$
P^{k}(M)=\arg \max _{P(M)}\left[-\frac{1}{\beta} \sum_{M} P(M) \ln P(M)+\sum_{M} P(M) \ln P\left(M \mid X, Y\left(T^{(k-1)}\right)\right)\right]
$$

under the constraint $\sum_{M} P(\mathbf{M}=M)=1$, where $\beta=\frac{1}{t}$, and $t$ is the temperature, which determines the annealing schedule. The annealing schedule is imposed to incorporate subjective information in estimating $P^{k}(M)$ [2].

To overcome the need of the EM algorithm for known component densities, we propose estimating $p_{a}\left(x_{i}, y_{i}\right)$ from the given data set at the current estimated transformation parameters. Note that we use Parzen window method [9] for nonparametric density function. However, these estimated joint density functions cannot be used in the EM algorithm. Instead, we evaluate the expected value of the objective function in the EM algorithm w.r.t. $p\left(x_{i}, y_{i}\right)$ which leads to the min step (see [2]):

$\underline{\text { Min Step }:} \quad T^{k}=\arg \min _{T} \sum_{a \in A} \frac{1}{N^{2}}\left(\sum_{i=1}^{N^{2}} P_{i}^{k}(a)\right) H_{a}(x, y)-H(y)$

which is joint conditional entropy $H(M, X \mid Y)$. The component density function for class $a, p_{a}(x, y)$, is estimated as the weighted sum of Gaussian kernels, $G_{\psi}(x)=(2 \pi)^{\frac{-n}{2}}|\psi|^{\frac{-1}{2}} \exp \left(-\frac{1}{2} x^{T} \psi^{-1} x\right)$, using the Parzen window method as follows:

$$
p_{a}^{k}(x, y) \approx \frac{1}{\sum_{\left(x_{i}, y_{i}\right) \in \mathbf{I}} P_{i}^{k}(a)} \sum_{\left(x_{i}, y_{i}\right) \in \mathbf{I}} P_{i}^{k}(a) G_{\Psi_{a}}\left(x-x_{i}, y-y_{i}\right)
$$


where, $P_{i}^{k}(a)=P^{k}\left(\mathbf{m}_{i}=a\right)$ is the probability that the $i$ th pixel in the portal image belongs to class $a$, estimated in the max step, equation (3), $\Psi_{a}$ is 2 -by-2 covariance matrix, which is assumed to be diagonal. Note that this assumption does not means that the random variables $\mathbf{x}$ and $\mathbf{y}$ are independent. $\mathbf{I}$, J denote sets of sizes $N_{I}$ and $N_{J}$, respectively, of pixels sampled at random from the portal image, $X$, and the DRR, $Y$. The joint entropy functions, which are the expected value of the log of the joint probability density functions, are approximated as statistical expectations using the Parzen window density estimates as follows. $H_{a}(x, y)$

$$
\approx\left(\frac{-1}{\sum_{w_{j} \in \mathbf{J}} P_{j}^{k}(a)}\right) \sum_{w_{j} \in \mathbf{J}} P_{j}^{k}(a) \ln \left(\frac{1}{\sum_{w_{i} \in \mathbf{I}} P_{i}^{k}(a)} \sum_{w_{i} \in \mathbf{I}} P_{i}^{k}(a) G_{\Psi_{a}}\left(w_{j}-w_{i}\right)\right)
$$

where $w_{i}=\left(x_{i}, y_{i}\right)$. The entropy of the DRRs, $H(y)$, is estimated as in Viola and Wells [25]. While MI assumes that pixels are i.i.d., we avoid this assumption by using mixture densities. We note that Studholm et. al. [24], register images with mutual information as a match measure while incorporating segmentation information on one of the images. However, the image was pre-hand segmented. Coordinate Descent Interpretation: The minimax entropy algorithm above is developed within a probabilistic framework. However, within the optimization framework the algorithm can be viewed as a coordinate descent approach which seeks to optimize a cost function by iterative estimation of the parameters along different coordinates. Let

$$
\begin{aligned}
F(\tilde{P}, T) & =-H(M, X \mid Y)+H(M) \\
& =\iint d X d Y \sum_{M} p(X, Y \mid M) \tilde{P}(M) \ln p(M, X \mid Y)-\sum_{M} \tilde{P}(M) \ln \tilde{P}(M)
\end{aligned}
$$

Note that $F(\tilde{P}, T)$ is a functional (function of function) which is to be optimized to estimate density function $\tilde{P}(M)$ and the parameters, T. Optimizing $F(\tilde{P}, T)$ using the coordinate descent approach leads to the following two steps: Step 1: $\tilde{P}^{k}(M)=\arg \max _{\tilde{P}} F\left(\tilde{P}, T^{k-1}\right)$, under constraint $\sum_{M} \tilde{P}^{k}(M)=1$ Step 2: $T^{k} \quad=\arg \max _{T} F\left(\tilde{P}^{k}, T\right)$

Step 1, where the energy functional $F(\tilde{P}, T)$ is being optimized to estimate $\tilde{P}(M)$, utilizing the transformation parameters $T^{k-1}$, is equivalent to the max step. Thus, estimation of the density function $\tilde{P}(M)$, a variational calculus problem within the optimization framework, is interpreted as maximum entropy estimation of a density function within the probabilistic framework. Step 2, where we optimize $F(\tilde{P}, T)$ to estimate $\mathbf{T}$, utilizing current estimates of $\tilde{P}(M)$, is equivalent to the min step as the marginal entropy term, $H(M)$, is independent of the parameters $\mathbf{T}$.

Utilizing Dual Portal Images: It is expected that utilizing another portal image, typically the left-lateral (LL) portal image acquired in the orthogonal direction, will greatly enhance the accuracy of the estimated pose. Thus, we extend the algorithm to utilize two portal images, AP and LL, for the estimation of the pose. Both the min step and the max step are modified to incorporate this new information. While estimating the segmentations of the two 
portal images, in the max step, we assume that the segmentations of the two portal images are statistically independent. Thus, in the max step for the 2portal case, $P^{k}\left(M_{A P}\right)$ and $P^{k}\left(M_{L L}\right)$, are estimated by repeating equation (4) for the two portal images separately. Note that though there are two portal images, whose segmentation is being estimated separately, there is only one set of transformation parameters, $\mathbf{T}$, is to be estimated. In the initial formulation of the algorithm, the optimal pose parameters are thus estimated as $\hat{T}^{k}=\arg \min _{T}\left[H\left(M_{A P}, X_{A P} \mid Y_{1}\right)+H\left(M_{L L}, X_{L L} \mid Y_{2}\right)\right]$ where $X_{A P}, X_{L L}$ denote the AP and the LL portal image respectively, and $Y_{1}, Y_{2}$ denote the DRRs obtained from the 3D CT data set in the AP and LL directions.

\section{Results}

In this section we evaluate the accuracy and robustness of the proposed minimax algorithm using both real and simulated data. A plexi-glass pelvic bone phantom is scanned to provide the 3D CT dataset. The phantom consists of real human pelvic bone encased in plexi-glass of density close to the density of softtissue. The phantom is then moved to the treatment room to obtain real portal images at the treatment energy $\mathrm{X}$-rays $(6 \mathrm{MV})$. The simulated portal images are obtained in the following fashion. First, the 3D CT voxel values are mapped from diagnostic energy values to the values at the treatment energy $\mathrm{X}$-rays using attenuation coefficient tables [15]. Second, the 3D CT data set is transformed by known transformation parameters. Third, the digitally reconstructed radiographs (DRRs) are rendered, using perspective projection, from the CT data set, both in the anterior-posterior (AP) and the left-lateral (LL) directions. Two different testing sets of simulated portal images are then generated from the resulting DRRs. To obtain the first set of simulated portal images, varying amounts of i.i.d. Gaussian noise are added to the DRRs. To obtain the second set of simulated portal images, the DRRs are blurred using blurring kernels of increasing width, which simulates the finite size of the radiation source, and low contrast and low sharpness of the real portal images. Since the true registration parameters are known for the simulated portal images, these datasets are used to study the accuracy and robustness of the algorithm under increasing noise and blur in the images.

Our previous work [1] suggested that the proposed algorithm is not robust to the estimation of the out-of-plane transformation parameters when using only single AP portal images. The second portal image would be expected to improve this robustness. The in-plane translations for the AP portal image consists of translations along the $\mathrm{X}$ and $\mathrm{Y}$ axes and the in-plane rotation is the rotation about the $\mathrm{Z}$-axis, $\theta_{X Y}$. For the lateral portal image, the in-plane translations are the translations of the 3D CT dataset along the $\mathrm{Y}$ and $\mathrm{Z}$ axis and the in-plane rotation is the rotation about the $\mathrm{X}$-axis, $\theta_{Y Z}$. Note that by using two portal images, the rotation about the $\mathrm{Y}$-axis, $\theta_{X Z}$, is the only out-of-plane parameter to be estimated. 


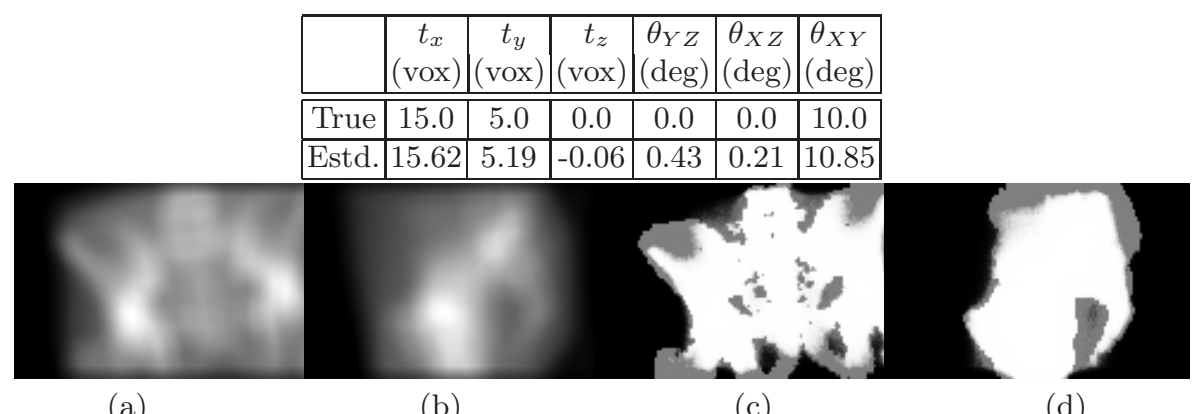

(a)

(b)

(c)

(d)

Fig. 1. (a) Simulated AP portal image. (b) Simulated left-lateral portal image. (c) Estimated segmentation of the AP portal image. (d) Segmentation of the LL portal image estimated by the algorithm. Estimated and the true parameters are shown in the table.

\subsection{Dual Simulated Portal Data}

The simulated portal images are obtained as explained above. The six transformation parameters to be estimated are denoted as $t_{x}, t_{y}$ and $t_{z}$ (along the $\mathrm{x}$-axis, $\mathrm{y}$-axis and $\mathrm{z}$-axis respectively) and the three rotations are denoted as $\theta_{Y Z}, \theta_{X Z}$ and $\theta_{X Y}$ (about the $\mathrm{x}$-axis, $\mathrm{y}$-axis and $\mathrm{z}$-axis respectively).

The simulated dual portal images are blurred using a uniform blurring kernel of width 11 to obtain the portal images shown in figure 1 (a), (b). Figure 1 (c), (d) show the corresponding segmentation of the portal images estimated by the algorithm. The table in the figure shows the true and the estimated parameters. Note that the estimated translations are within 1 voxel of the true values, even in the presence of a blur of 11 pixels. The estimates of the rotation parameters are within $0.5^{\circ}$, on average, of the true values.

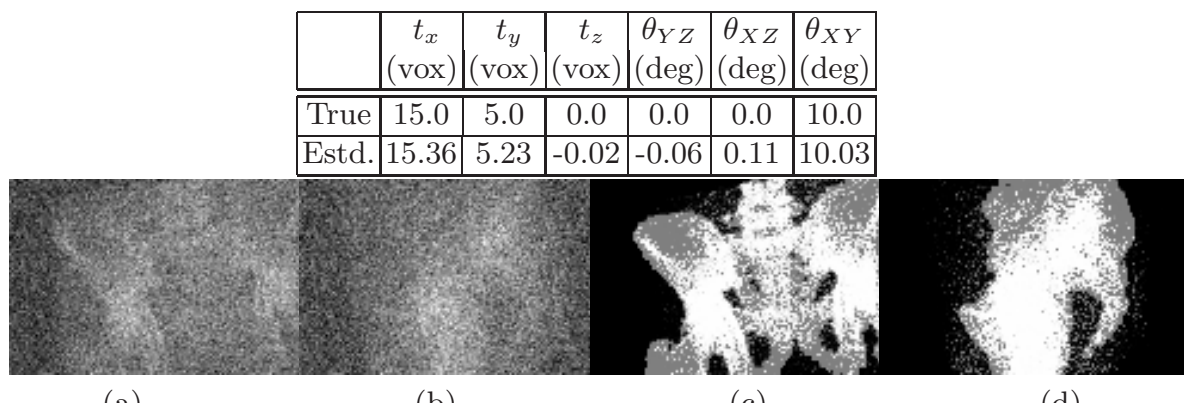

(a)

(b)

(c)

(d)

Fig. 2. Simulated portal images with noise. (a) AP with std 30.0 (b) Left-lateral with std 30.0 Estimated segmentation of (c) AP portal image. (d) LL portal image. The table show the true and the parameters estimated by the algorithm. 
Figure 2 (a), (b) shows the simulated portal images with Gaussian noise of standard deviation $=30.0$. The dynamic range of the pixel intensities was 255 . Note again the accuracy of the parameters estimated by the algorithm. Figure 2 (c), (d) shows the segmentation of the portal images as estimated by the algorithm.

Performance under varying initializations: Figure 3 (a) and (b) shows the graphs of error in estimated parameters for varying amounts of rotational and translational setup variations, in the presence of Gaussian noise of standard deviation $(\sigma)=20.0$ in the simulated portal images. To obtain these graphs, first, the 3D CT dataset is transformed by a known amount and, AP and LL DRRs are obtained. Then i.i.d. Gaussian noise of $\sigma=20.0$ is added to the DRRs to obtain the simulated portal images. For the graph labeled $\theta_{Y Z}$, only the parameter $\theta_{Y Z}$, which denotes rotation about the $\mathrm{X}$-axis, is varied to obtain the DRRs. All other parameters are kept fixed at the true values. The 3D CT is then reset to its untransformed position and the algorithm is run to estimate $\mathbf{T}$. The error in the estimated parameter is then plotted. The graphs show that, for this dataset, the algorithm could estimate the rotation angles up to $50^{\circ}$ accurately. For the translations, the estimates for the three translations were accurate up to 25 or more voxels. These figures also show that either the algorithm is quite accurate in estimating the parameters or it breaks down completely, that is, the estimated parameters are totally different than the true parameters. This shows that the algorithm gets trapped into a local minimum if the global minimum is very far from the initial starting position.

Performance under varying noise: Figures 3 (c) and (d) show the performance of the algorithm under increasing noise. The AP and LL portal images, for example for the graph labeled $\theta_{X Y}$, are obtained by first rotating the 3D CT data by $15^{\circ}$ about the $\mathrm{Z}$-axis and then rendering the DRRs both in the AP and the LL directions. A varying amount of noise is then added to the DRRs to obtain the simulated portal images. The 3D CT data set is then initialized to its undeformed position and the algorithm is run to estimate $\mathbf{T}$. The graph shows the error in estimated $B d T$ for various amounts of noise. Similarly, for the graphs labeled $\theta_{Y Z}, \theta_{X Z}, t_{x}, t_{y}, t_{z}$, the $3 \mathrm{D} \mathrm{CT}$ data set was transformed by $30^{\circ}, 25^{\circ}, 20$ voxels, 20 voxels and 15 voxels respectively to obtain the DRRs.

\subsection{Performance on Actual Patient Treatment Data}

Figure 4 shows the results of running the proposed algorithm on real patient data. Figures 4 (a) and (b) show histogram equalized AP and LL portal images, respectively. The DRRs projected through the 3D CT data in its original pose are shown in the figures 4 (c) and (d). Running the algorithm estimates a new pose of the 3D CT dataset, which differs from the original pose by $\theta_{X Y}=3.2^{\circ}, \theta_{Y Z}=$ $2.92^{\circ}, \theta_{X Z}=1.93^{\circ}, t_{x}=3.53$ voxels, $t_{y}=12.5$ voxels and $t_{x}=13.54$ voxels. The DRR projections in the new pose are shown in the figures 4 (e) and (f). Segmentations of the AP and LL portal images, estimated by the algorithm, 
are shown in the figures $4(\mathrm{~g})$ and $(\mathrm{h})$, respectively. Due to poor quality of these digitized portal film images, the segmentation step was initialized manually in several regions to highlight the background. To assess the accuracy of the estimated pose of the 3D CT data set, contours are hand drawn on the portal images, matching visible features. These contours are then mapped onto the DRRs, in figures 4 (c), (d), (e), (f) undeformed. Note that the contours are used only to visually assess the goodness of the estimated pose. The contours match closely to the features in DRRs obtained at the pose estimated by the algorithm, although there is some remaining error, perhaps resulting from error in $\theta_{X Z}$ (out-of-plane rotation for both views).

\section{Discussion and the Future Work}

In this work we extended our algorithm [1] to utilize dual portal images to estimate the transformation parameters, note the performance of the algorithm utilizing real patient data, present experiments which demonstrate the extent of parameters algorithm can estimate, demonstrate the robustness of the algorithm under increasing noise, and present a coordinate descent interpretation of the algorithm.

Our future research includes algorithm speed-up, validation of the accuracy and robustness of the algorithm, especially in comparison to the mutual information based registration and the ridge based algorithm [11]. Effects of artifacts in the portal images, like air bubbles, unstable features, like movement of femurs w.r.t. pelvic bone and portal images with exposures only through the limited treatment field require further study. The inclusion of edges and whole boundary information will likely lead to more accurate results. Thus, we will extend our algorithm to incorporate such information into the same framework.

\section{References}

1. R. Bansal, L. Staib, Z. Chen, A. Rangarajan, J. Knisely, R. Nath, and J.S. Duncan. A novel approach for the registration of 2D portal and 3D CT images for treatment setup verification in radiotherapy. Medical Image Computing and ComputerAssisted Intervention (MICCAI'98), LNCS-1496:1075-1086, 10-12 October 1998. 154, 159, 162

2. R. Bansal, L. Staib, et al. A minimax entropy registration framework for patient setup verification in radiotherapy: Evaluation and comparisons. Technical Report YALE-IPAG-TR-1999-01, Dept. of Electrical Engineering and Diagnostic Radiology, Yale University, Jan 1999. 156, 157

3. Martin Berger and Guido Gerig. Motion Measurments in Low-Contrast X-ray Imagery. In W.M. Wells et al., editors, Medical Image Computing and ComputerAssisted Intervention -MICCAI'98, volume 1496 of LNCS, pages 832-841, 1998. 154

4. R. M. Bevensee. Maximum Entropy Solutions to Scientific Problems. P T R Prentice Hall, 1993. 157 
5. J. Bijhold et al. Radiation field edge detection in portal images. Phys. Med. Biol., 36(12):1705-1710, 1991. 154

6. A. Collignon, F. Maes, et al. Automated multimodality image registration using information theory. Info. Proc. in Med. Imaging (IPMI), pages 263-274, 1995. 156

7. A. P. Dempster, N. M. Laird, and D. B. Rubin. Maximum likelihood from incomplete data via EM algorithm. J. Royal Statistical Soc., Ser. B, 39:1-38, 1977. 155, 156

8. L. Dong and A. L. Boyer. An image correlation procedure for digitally reconstructed radiographs and electronic portal images. Int. J. Radiation Oncol. Biol. Phys., 33(5):1053-1060, 1995. 154

9. R. O. Duda and P. E. Hart. Pattern Classification and Scene Analysis. John Wiley \& Sons, 1973. 157

10. D. S. Fritsch et al. Core-based portal image registration for automatic radiotherapy treatment verification. Int. J. Radiation Oncol. Biol. Phys., 33(5):1287-1300, 1995. 154

11. K. Gilhuijs. Automated verification of radiation treatment geometry. PhD thesis, Univ. of Amsterdam, Radiotherapy dept., the Netherlands, 1995. 154, 162

12. K. Gilhuijs et al. Interactive three dimensional inspection of patient setup in radiation therapy using digital portal images and computed tomography data. Int. J. Rad. Oncol. Biol. Phys., 34(4):873-885, 1996. 154

13. J. Hanley et al. The effects of out-of-plane rotations on two dimensional portal image registration in conformal radiotherapy of the prostate. Int. J. Radiation Oncology Biol. Phys., 33(5):1331-1343, 1995. 153, 154

14. D. H. Hiristov and B. G. Fallone. A gray-level image alignment algorithm for registration of portal images and digitally reconstructed radiographs. Med. Phys., 23(1):75-84, Jan 1996. 154

15. J. H. Hubble. Photon Cross Sections, Attenuation Coefficients, and Energy Absorption Coefficients From $10 \mathrm{KeV}$ to $100 \mathrm{GeV}$. Nat. Stand. Ref. Data. Ser., Nat. Bur. Stand. (U.S.), August 1969. 159

16. E. T. Jaynes. Prior probabilities. In R. D. Rosenkrantz, editor, E. T. Jaynes: Papers on probability, statistics and statistical physics, volume 158, pages 114-130. D. Reidel Publishing Company, Boston. USA, 1983.

17. S. Kirkpatrick et al. Optimization by simulated annealing. Science, 220:671-680, 1983. 157

18. S. Lavallee, R. Szeliski, and L. Brunie. Anatomy-based registration of three-dimensional medical images, range images, $\mathrm{X}$-ray projections, and threedimensional models using octree-splines. In Russell H. Taylor et al., editors, Computer-Integrated Surgery: Technology and Clinical Applications, pages 115143. The MIT Press, Cambridge, Massachusetts, 1996. 154

19. L. Lemieux et al. A patient-to-computed-tomography image registration method based on digitally reconstructed radiographs. Med. Phys., 21(11):1749-1760, November 1994. 154

20. K. W. Leszczynski, S. Loose, and S. Boyko. An image registration scheme applied to verification of radiation therapy. The British Journal of Radiology, 71(844):413426, 1998. 154

21. H. Meertens, J. Bijhold, and J. Strackee. A method for the measurement of field displacement errors in digital portal images. Phy. Med. Biol., 35(3):299-323, 1990. 154

22. C. D. Mubata, A. M. Bidmead, et al. Portal imaging protocol for radical doseescalated radiotherapy treatment of prostate cancer. Int. J. Radiation Oncol. Biol. Phys., 40(1):221-231, 1998. 154 
23. W. D. Neve, F. Heuvel, et al. Interactive use of on-line portal imaging in pelvic radiation. Int. J. Radiation Oncology Biol. Phys., 25:517-524, 1993. 154

24. C. Studholme et al. Incorporating connected region labelling into automated image registration using mutual information. In Proc. of MMBIA'96, pages 23-31, 1996. 158

25. P. Viola and W. M. Wells. Alignment by maximization of mutual information. Fifth Int. Conf. on Computer Vision, pages 16-23, 1995. 156, 158

26. J. Weese et al. 2D/3D registration of pre-operative ct images and intra-operative $\mathrm{x}$-ray projections for image guided surgery. In H.Lemke et al., editors, Comp. Assist. Rad. and Surg., pages 833-838. 1997. 154 


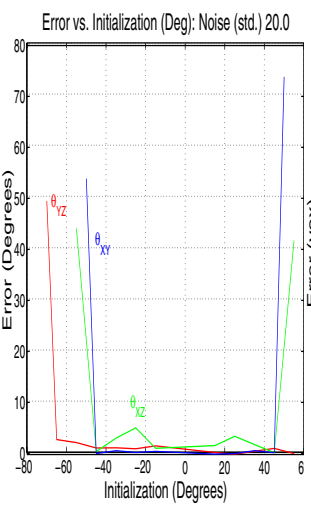

(a)

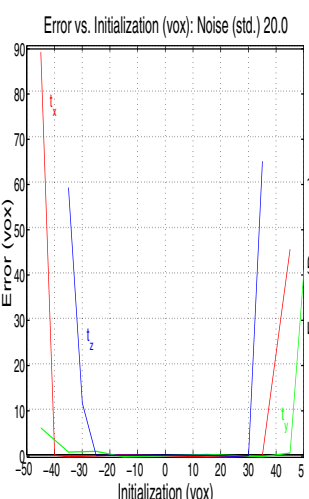

(b)

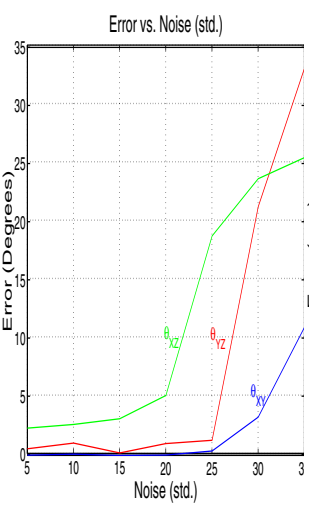

(c)

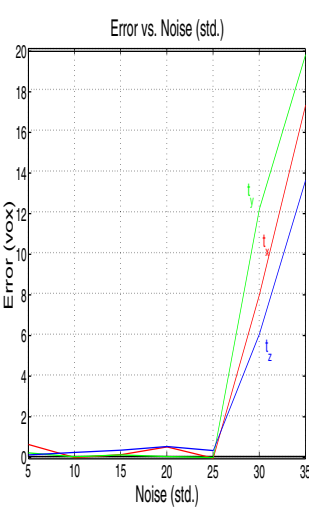

(d)

Fig. 3. (a) Error in estimated rotation angles. (b) Error in estimated translation. (c) Error in estimated angles with increasing noise. (d) Error in estimated translation with increasing noise.

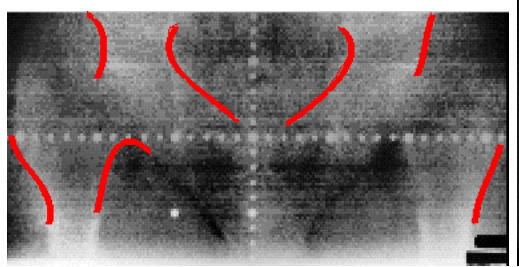

(a)

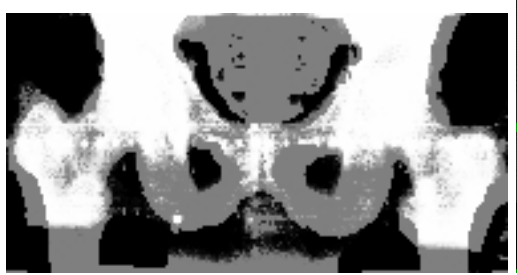

(g)

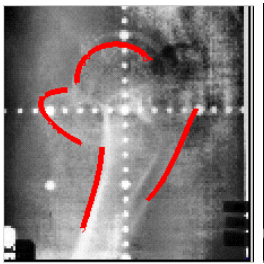

(b)

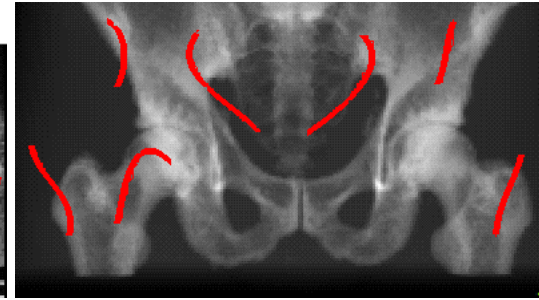

(c)

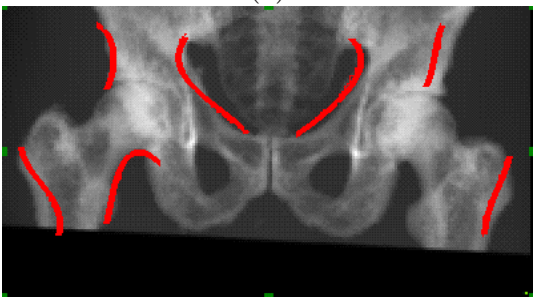

(e)

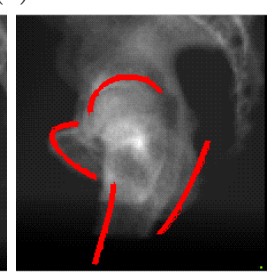

(f)

Fig. 4. Recovery of setup variation using actual patient data. (a,b) Portal images, (c,d) DRR's of 3D CT in original pose, $(e, f)$ DRR's in corrected pose, $(g, h)$ implicit segmentation of portals. 